\title{
A Non-side-looking Range Compensation Method for MIMO Radar
}

\author{
Yang Liu ${ }^{+}$, Yong-Shun Zhang, Qi-Chao Ge, Zhi-Hui Li \\ Air and Missile Defense College, Air Force Engineering University, Xi'an 710051, China
}

\begin{abstract}
The loss for degree of system freedom and mismatch of prior information based on registration based compensation method will result in a decline in the computation performance. In order to solve the above problems, an improved method is proposed where sparse recovery theory is applied instead of the sub-aperture smoothing operation. Firstly, it combines sparse recovery algorithm with MIMO radar system whose single frame data are equivalent to multi frame data of the phased array. Then the super resolution clutter space-time spectrum is gotten by utilizing the sparse recovery. After that the transition covariance matrix is calculated. Finally, the clutter covariance matrix is reconstructed by Capon spectrum. Compared with the conventional RBC (Registration Based Compensation) method, the method proposed doesn't depend on sub-aperture smoothing operation and prior information. In addition, it is also steadier when the sensor error exists. The experimental simulation demonstrates the correction and feasibility of this method.
\end{abstract}

Keywords: MIMO radar; STAP; range dependence; sub-aperture smoothing; sparse recovery.

\section{Introduction}

STAP (Space-Time Adaptive Processing) technology has a good effect on clutter suppression and strong active jamming. Meanwhile it improves the detection performance of ground moving targets [1-2]. The estimation accuracy of clutter covariance matrix directly determines the merits and demerits of clutter suppression performance of STAP. In the side-looking airborne radar case, we can use diagonal loading to the training sample unit on both sides of the unit to be detected to get the clutter covariance matrix. It makes the adaptive filter achieve optimal performance and the training sample unit is detected to meet the requirements of IID (Independent Identically Distributed). Once the radar in the non-side-looking case, the Doppler frequency of ground clutter produces serious distance dependence, which results in the training sample unit will never satisfy IID condition. All of these make the estimated clutter covariance matrix seriously curve. Thereby the performance of clutter suppression by STAP is also affected [3-5]. For short-range clutter, this situation is particularly prominent.

To solve above problems, this paper proposes a distance compensation method based on sparse recovery [6-8] theory for airborne MIMO radar system. It is called M-SRBC method. Firstly, the single frame data in different range units of MIMO radar are equivalent to multi frame data of phased array radar. Secondly, it makes sparse process to the sparse clutter data of different range units, which discretizes the spatial domain and Doppler domain into many resolution cells and structures super complete basis matrix. Then it makes use of sparse recovery theory to obtain super resolution clutter spectrum and calculate the transitional covariance matrix. Finally it reconstructs the estimated clutter covariance matrix by the Capon, and obtains the transformation matrix by eigenvalue decomposition. The simulation results show that the M-SRBC method can effectively avoid the influence of the different distance unit inconsistency, which causes the clutter spectrum broadening. Meanwhile it solves the problem of clutter range compensation performance declining and keeps a good robustness when array error exists.

\section{Clutter Model and Signal Model}

\footnotetext{
+ Corresponding author.

E-mail address: liuyang77118@163.com.
} 
The number of transmitting array elements of the MIMO radar, the number of receiving array elements and the number of pulses are respectively $M, N, K$; The distance between the transmitting array elements and the receiving array elements are respectively $d_{T}$ and $d_{R}$.

The airborne MIMO radar clutter data in the $l$ th distance loop are divided into several blocks. Then the airborne non-side-looking radar meets the following spatial coupling relationship:

$$
\begin{aligned}
f_{D, i}\left(\theta_{i}, \varphi_{i}\right) & =\frac{2 V}{\lambda} \cos \varphi_{i} \cos \left(\theta_{i}+\alpha\right) \\
f_{S, i} & =\cos \varphi_{i} \cos \theta_{i}
\end{aligned}
$$

Where $\lambda$ is the emission wavelength, $V$ is the aircraft speed, $\alpha$ is the angle between speed and antenna array; $f_{D, i}$ and $f_{S, i}$ are respectively the Doppler frequency and spatial frequency corresponding to the ith clutter block; $\theta_{i}$ and $\varphi_{i}=\arcsin \left(H / R_{l}\right)$ are the azimuth angle and elevation angle in the ith clutter block.

According to the space-time coupling relationship of airborne MIMO radar, we can see that the Doppler frequency and spatial frequency have following relations:

$$
\left[\frac{f_{D, i}}{f_{D, m}}\right]^{2}+f_{S, i}{ }^{2}-2 \frac{f_{D, i}}{f_{D, m}} f_{S, i} \cos \alpha=\left(\frac{\sqrt{R_{l}^{2}-H^{2}}}{R_{l}} \sin \alpha\right)^{2}
$$

Where $f_{D, m}=2 V / \lambda$ is the maximum Doppler frequency of clutter; $R_{l}$ is the slant distance of the radar relative to the $l$ th distance gate.

The echo data can be expressed as the superposition of several discrete clutter blocks on the range gate:

$$
\boldsymbol{X}_{l}=\sum_{i=1}^{G} \sigma_{i} \boldsymbol{S}_{i}\left(w_{T}, w_{S}\right) \in \mathbb{C}^{M N K \times 1}
$$

Where $G$ is the number of discrete clutter blocks, $\sigma_{i}$ is the scattering coefficient of the $i$ th clutter block, $S_{i}\left(w_{T}, w_{S}\right)$ is the transmitting-receiving-time three-dimensional steering vector corresponding to the $i$ th clutter block, $w_{S}$ and $w_{T}$ are respectively the spatial angular frequency and time angular frequency.

The echo data of the $l$ th range unit is equivalent to the following matrix form:

$$
\boldsymbol{X}_{l}=\left[\boldsymbol{x}_{l, 1}, \boldsymbol{x}_{l, 2}, \cdots, \boldsymbol{x}_{l, m}, \cdots, \boldsymbol{x}_{l, M}\right] \in \mathbb{C}^{N K \times M}
$$

Where $\boldsymbol{x}_{l, m}(m=1,2, \ldots, M)$ is the echo data corresponding to the $m$ th emission waveform.

$$
\boldsymbol{S}_{i}\left(w_{T}, w_{S}\right)=\boldsymbol{S}_{T, i}\left(w_{T, i}\right) \otimes \boldsymbol{S}_{S_{t}, i}\left(w_{S, i}\right) \otimes \boldsymbol{S}_{S_{r}, i}\left(w_{S, i}\right)
$$

Where $\boldsymbol{S}_{S_{t}, i}\left(w_{S, i}\right), \boldsymbol{S}_{S_{r}, i}\left(w_{S, i}\right)$ and $\boldsymbol{S}_{T, i}\left(w_{T, i}\right)$ are respectively the emission space steering vector, the received spatial steering vector and the time domain steering vector corresponding to the $i$ th clutter block, $\otimes$ is the Kronecker product.

$$
\left\{\begin{array}{l}
\boldsymbol{S}_{T, i}\left(w_{T, i}\right)=\left[\begin{array}{llll}
1 & \mathrm{e}^{\mathrm{j} w_{T, i}} & \cdots & \mathrm{e}^{\mathrm{j}(K-1) w_{T, i}}
\end{array}\right]^{T} \\
\boldsymbol{S}_{S_{t}, i}\left(w_{S_{t}, i}\right)=\left[\begin{array}{llll}
1 & \mathrm{e}^{\mathrm{j} w_{S, i}} & \cdots & \mathrm{e}^{\mathrm{j} \mu(M-1) w_{S, i}}
\end{array}\right]^{T} \\
\boldsymbol{S}_{S_{r}, i}\left(w_{S_{r}, i}\right)=\left[\begin{array}{llll}
1 & \mathrm{e}^{\mathrm{j} w_{S, i}} & \cdots & \mathrm{e}^{\mathrm{j}(N-1) w_{S, i}}
\end{array}\right]^{T}
\end{array}\right.
$$

Where $w_{T, i}=2 \pi f_{D, i} / f_{p r f}, w_{S, i}=2 \pi d_{R} f_{S, i} / \lambda ; f_{p r f}$ is the pulse repetition frequency, $\mu$ is the ratio between the transmitting element distance and the receiving element distance.

\section{Improved Method}

The clutter data of each distance ring is formed by the superposition of many space-time three dimensional steering vectors. Therefore the clutter data in the $l$ th distance ring can be expressed as: 


$$
\boldsymbol{X}_{l}=\sum_{j=1}^{N_{S} N_{D}} \gamma_{j} \boldsymbol{S}_{j}\left(w_{T, j}, w_{S, j}\right)=\boldsymbol{\Psi}_{l} \boldsymbol{\alpha}_{l}
$$

Where $\gamma_{j}$ is the plural amplitude corresponding to the $j$ th space-time two dimensional steering vector of the clutter is represented; $\boldsymbol{\alpha}_{l}$ is the frequency distribution of the clutter data in the $l$ th distance ring, $\boldsymbol{\Psi}_{l}$ is over-complete matrix size $M N K \times N_{S} N_{D}$ :

$$
\begin{aligned}
& \alpha_{l}=\left[\alpha_{l, 1}, \alpha_{l, 2}, \cdots, \alpha_{l, N_{S} N_{D}}\right] \\
& \Psi_{l}=\left[\begin{array}{c}
S_{1}\left(w_{t, 1}, w_{s, 1}\right)^{\mathrm{T}} \\
S_{2}\left(w_{T, 2}, w_{S, 2}\right)^{\mathrm{T}} \\
\vdots \\
\boldsymbol{S}_{j}\left(w_{T, j}, w_{S, j}\right)^{\mathrm{T}} \\
\vdots \\
\boldsymbol{S}_{N_{S} N_{D}}\left(w_{T, N_{S} N_{D}}, w_{S, N_{S} N_{D}}\right)^{\mathrm{T}}
\end{array}\right]^{\mathrm{T}}
\end{aligned}
$$

Where $N_{S}=\rho_{S} N$ and $N_{D}=\rho_{D} K$ are the resolution cells obtained by quantifying the spatial frequency and the Doppler frequency; $\rho_{S}$ and $\rho^{D}$ are the quantization scale of spatial frequency and Doppler frequency, in order to obtain accurate space-time distribution characteristics, the values greater than 1 in general.

The space-time spectrum $\alpha_{l}$ can be obtained in the case of $X_{l}$ and $\Psi_{l}$ are known. Because of the matrix $\Psi_{l}$ is over-complete $\left(N_{S} N_{D} \gg M N K\right)$, so we need to solve the underdetermined equation. According to the sparse recovery theory, when the vector is sparse, the underdetermined equation can be solved efficiently:

$$
\left\{\begin{array}{c}
\hat{\alpha}_{l}=\min \left\|\alpha_{l}\right\|_{1} \\
\text { s.t. }\left\|\boldsymbol{X}_{l}-\boldsymbol{\Psi}_{l} \boldsymbol{\alpha}_{l}\right\|_{2} \leq \varepsilon
\end{array}\right.
$$

Where $\hat{\boldsymbol{\alpha}}_{l}$ is the space-time spectrum estimation, \|\|$_{p}$ is the $L_{p}$ norm, $\varepsilon$ is the matching error.

At last, the sparse covariance matrix is calculated by the space-time spectrum estimation $\hat{\alpha}_{l}$ and the over-complete matrix $\Psi_{l}$ of different distance units:

$$
\tilde{\boldsymbol{R}}_{l}=\sum_{i=1}^{N_{S} N_{D}}\left|\hat{\alpha}_{l}(i)\right|^{2} \boldsymbol{\Psi}_{i, l} \boldsymbol{\Psi}_{i, l}^{H}+\boldsymbol{I}
$$

Where $\hat{\alpha}_{l}(i)$ is the $i$ th element in the space-time spectrum $\hat{\alpha}_{l}$ of the $l$ th distance unit, $\Psi_{i, l}$ is the $i$ th column data in over-complete matrix $\Psi_{l},(\bullet)^{H}$ is conjugate transpose, $\boldsymbol{I}$ is zero matrix size $M N K \times N_{S} N_{D}$.

We choose $P$ discrete points from the angle-Doppler frequency distribution curve on the $l$ th distance $\operatorname{ring}(P>2 M N K)$. Because of the clutter spectrum intensity can be directly reflected by Capon, so the scattering coefficient at the $t$ th scattering point can be shown by $P\left(w_{S, t}, w_{T, t}\right)$, then the covariance matrix estimation without compensation is reconstructed:

$$
\begin{gathered}
P\left(w_{S, t}, w_{T, t}\right)=\frac{1}{\tilde{\boldsymbol{W}}^{H}\left(w_{S, t}, w_{T, t}\right) \tilde{\boldsymbol{R}}_{l}^{-1} \tilde{\boldsymbol{W}}\left(w_{S, t}, w_{T, t}\right)} \\
\hat{\boldsymbol{R}}_{l}=\sum_{t=1}^{P}\left|P\left(w_{S, t}, w_{T, t}\right)\right|^{2} \boldsymbol{W}\left(w_{S, t}, w_{T, t}\right) \boldsymbol{W}^{H}\left(w_{S, t}, w_{T, t}\right)
\end{gathered}
$$

Where $w_{S, t}$ and $w_{T, t}$ are the spatial angular frequency and time angular frequency on the angle-Doppler frequency distribution curve of the $t$ th scattering points; $\boldsymbol{W}\left(w_{S, t}, w_{T, t}\right)$ is the sparse space-time three-dimensional steering vector; $\boldsymbol{W}\left(w_{S, t}, w_{T, t}\right)$ is the space-time three-dimensional steering vector on the space-time plane.

The $L$ th training sample unit is designated as the unit to be detected, then the clutter covariance matrix of the $L$ th distance unit is reconstructed. Now it is necessary to transform the clutter statistical properties of the $l$ th distance to be the same as unit the unit to be detected:

$$
\min _{\boldsymbol{T}_{l}}\left\|\boldsymbol{T}_{l} \hat{\boldsymbol{R}}_{l} \boldsymbol{T}_{l}^{H}-\hat{\boldsymbol{R}}_{L}\right\|_{2}
$$


Where \|\|$_{2}$ is the 2-norm of the matrix. Apply eigenvalue decomposition operation on $\hat{\boldsymbol{R}}_{l}$ and $\hat{\boldsymbol{R}}_{L}$ :

$$
\begin{gathered}
\hat{\boldsymbol{R}}_{l}=\boldsymbol{V}_{l} \boldsymbol{\Lambda}_{l} \boldsymbol{V}_{l}^{H}=\left(\boldsymbol{V}_{l} \boldsymbol{\Lambda}_{l}^{1 / 2}\right)\left(\boldsymbol{V}_{l} \boldsymbol{\Lambda}_{l}^{1 / 2}\right)^{H} \\
\hat{\boldsymbol{R}}_{L}=\left(\boldsymbol{V}_{L} \boldsymbol{\Lambda}_{L}^{1 / 2}\right)\left(\boldsymbol{V}_{L} \boldsymbol{\Lambda}_{L}^{1 / 2}\right)^{H}
\end{gathered}
$$

Where $V_{l}$ and $V_{L}$ are the feature vector matrix, $\Lambda_{l}$ and $\Lambda_{L}$ are the corresponding eigenvalue matrix. The space-time transformation matrix is:

$$
\boldsymbol{T}_{l}=\boldsymbol{V}_{L} \boldsymbol{\Lambda}_{L}^{1 / 2} \boldsymbol{\Lambda}_{l}^{-1 / 2} \boldsymbol{V}_{l}^{H}
$$

In order to make the clutter characteristics of the training unit to be consistent with the unit to be detected, the space-time transformation matrix $\boldsymbol{T}_{l}$ is used to the radar echo data to achieve the purpose of clutter range dependence compensation:

$$
\tilde{\boldsymbol{X}}_{l}=\boldsymbol{T}_{l}^{H} \boldsymbol{X}_{l}
$$

\section{Experimental Simulation}

Parameter settings: Height of aircraft flight is $6 \mathrm{~km}$, speed is $140 \mathrm{~m} / \mathrm{s}$, wavelength is $0.23 \mathrm{~m}$, pulse repetition frequency is $2434.8 \mathrm{~Hz}$, maximum detection range is $800 \mathrm{~km}$, transmitting and receiving array element number are all 6, pulse number in one coherent processing interval is 6 , the array element distance is $0.115 \mathrm{~m}$, azimuth of beam center is $90^{\circ}, \mathrm{CNR}$ is $60 \mathrm{~dB}$. In order to simplify the problem, the distance ambiguity is not considered.

In the experiment, the 70 units before and after the test unit $(l=121)$ were taken as the training sample unit. The degree of spatial frequency discretization and the Doppler frequency discretization in the sparse recovery process are all $8, \rho_{S}=\rho_{D}=8$; the yaw angle is $3^{\circ}$.

\subsection{Clutter Power Spectrum Estimation}

In the cases of $5 \%$ array error, the real clutter power spectrum, the clutter power spectrum without compensation, the spectrum compensation method of phased array RBC and the spectrum compensation method of M-SRBC are compared. The results are shown in figure 1-2.
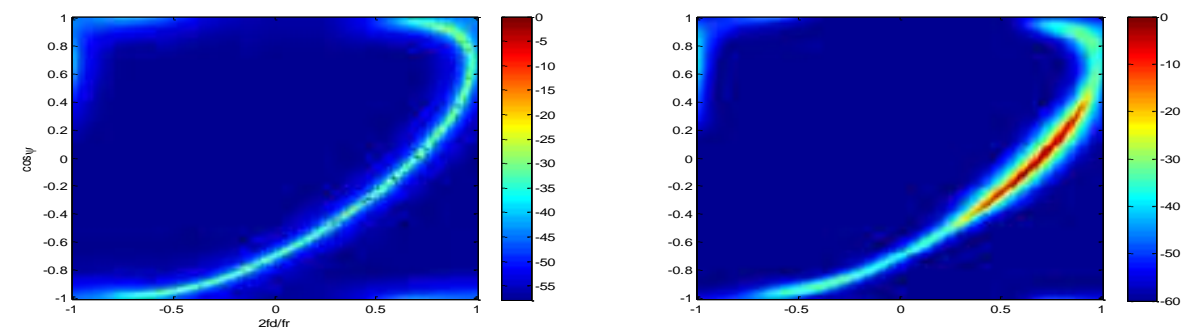

Fig. 1: The real clutter spectrum and the clutter spectrum without compensation

By figure 1 analysis, in the case of non-side-looking, the short-range clutter has serious range dependence. The method of diagonal loading the training sample units on both sides of the unit to be detected has little effect, each clutter range unit has a serious aliasing phenomenon, the clutter power spectrum obviously broadening compared with the real clutter spectrum.


Fig. 2: Phased array RBC in array error case and MIMO RBC in array error case

Comprehensive analysis of figure 2 shows that when there is priori information mismatch and array error, compared to the phased array RBC method, the M-SRBC method gives a better compensation performance. Meanwhile in the array error case, the M-SRBC method is little affected, although the system stability of the two methods are all decreased.

\subsection{Clutter Improvement Factor}


The detection performance of the system can be described by IF (Improvement Factor), which is the ratio of the output SCNR (Signal to Clutter-plus-Noise Ratio) and the input SCNR. Figure 3 respectively show the comparison results of improvement factor and the local enlarged image of the phased array RBC, phased array sparse RBC and M-SRBC method.
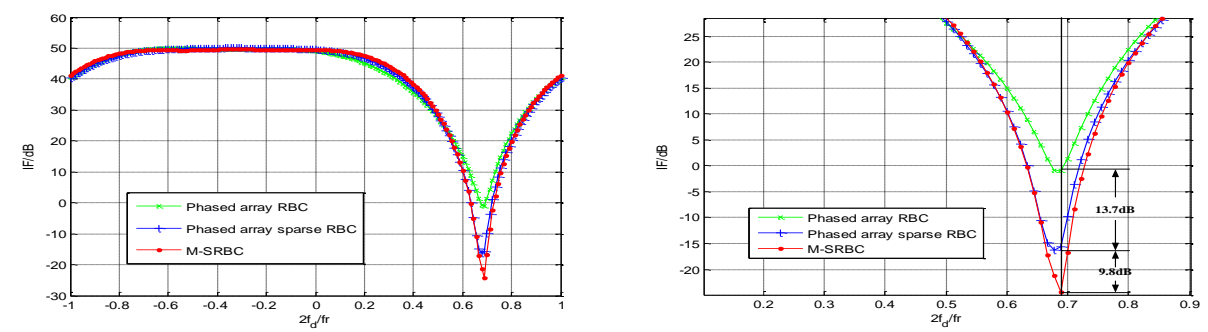

Fig. 3: Improvement factor image of each method and Local enlarged image of IF

By figure 3 analysis, three methods all have certain inhibition effect on clutter, but compared with the other two methods, M-SRBC method can form a deeper notch in the main lobe direction, the clutter suppression capability is more obvious. Compared with the phased array RBC method [9] and the phased array sparse RBC method [10], the clutter suppression performance of the M-SRBC method respectively increase $23.5 \mathrm{~dB}$ and $9.8 \mathrm{~dB}$.

\section{Conclusion}

MIMO radar RBC method based on sparse recovery is proposed in which the single frame data of MIMO radar is equivalent to multi frame data of phased array. It uses sparse recovery instead of sub-aperture smoothing to preprocess clutter echo data. The clutter covariance matrix reconstruction is estimated by the Capon spectrum. At last the clutter range dependence compensation is achieved by the transformation matrix. Research shows that, compared with the other two methods, the M-SRBC method with full-aperture smoothing is no loss of freedom, which avoids the loss of sub-aperture and the priori information mismatch problem. In the case of array errors, it can also achieve distance dependent compensation. And clutter suppression performance is better.

\section{References}

[1]. B Louis Fertig. Analytical expressions for space-time adaptive processing performance. IEEE Transactions on Aerospace and Electronic Systems, 2015,51(1):42-53.

[2]. R Klemm. The applications of space-time adaptive processing. London, UK: IEE, 2004.

[3]. M Shen, X Meng and L Zhang. Efficient adaptive approach for airborne radar short-range clutter suppression. IET Radar, Sonar \& Navigation, 2012, 6(9):900-904.

[4]. T Wang, J X Wu. Range-dependent clutter suppression approach for non-side-looking airborne radar based on orthogonal waveforms. IET Radar, Sonar \& Navigation, 2015,9(2):210-220.

[5]. S Beau, S Marcos, Range dependent clutter using rang-recursive space-time adaptive processing algorithms. Signal Processing, 2010,90(1):57-68.

[6]. K Sun, H Zhang, G Li, et al. STAP via sparse recovery of clutter spectrum. Acta Electronica Sinica, 2011, 39(6): 1389-1393.

[7]. $\mathrm{N} \mathrm{Hu}, \mathrm{Z} \mathrm{F} \mathrm{Ye} \mathrm{and} \mathrm{D} \mathrm{Y} \mathrm{Xv,} \mathrm{et} \mathrm{al.} \mathrm{A} \mathrm{sparse} \mathrm{recovery} \mathrm{algorithm} \mathrm{for} \mathrm{DOA} \mathrm{estimation} \mathrm{using} \mathrm{weighted} \mathrm{subspace} \mathrm{fitting.}$ Signal Processing, 2012,92(10):2566-2570.

[8]. H Yu, G H Lu, H L Zhang. Adaptive sparse recovery of moving targets for distributed MIMO radar. Advanced Materials Research, 2014,933:450-455.

[9]. F D Lapierre, J G Verly. Registration-based range dependence compensation for bistatic STAP radar. EURASIP JASP, 2005, 85-98.

[10]. K Sun, H D Meng, F D Lapierre, X Q Wang. Registration-based compensation using sparse representation in conformal-array STAP. Signal Processing, 2011, 91(10):2268-2276. 